\title{
Weg von der Agenturtheorie? Aber was dann?
}

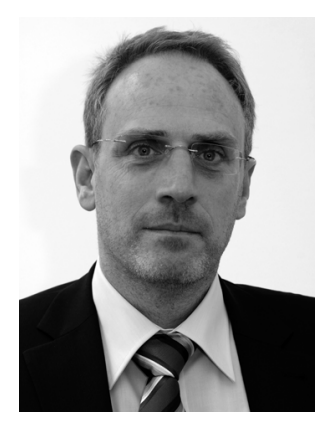

Peter Walgenbach

Corporate Governance, Agenturtheorie, „Shareholder Primacy“Modell, „Board Primacy“-Modell

Corporate governance, agency theory, shareholder primacy, board primacy

Ansetzend an der Kritik von Egon Franck, dass die Agenturtheorie nicht die institutionelle Wirklichkeit der modernen Kapitalgesellschaft abbildet, wird die Kritik der Agenturtheorie fortgeführt. Anschliessend werden mögliche Forschungsfelder für die betriebswirtschaftliche Forschung abgeleitet.

Based on Egon Franck's argument that agency theory does not correspond to the institutional reality of the modern corporation, the critique of agency theory will be continued. Subsequently, implications for future research in the field of organization and management studies will be developed.

„Ist es an der Zeit, die Aktionärsrechte zu stärken?“, fragt Egon Franck (2011) in seinem sehr lesenswerten Beitrag. Nach über 30 Jahren der Dominanz der Agenturtheorie in der nationalen und internationalen Corporate-Governance-Diskussion lautet die reflexhafte Antwort auf die gestellte Frage: Wenn nicht die Aktionärsrechte, was denn dann?

\section{Die Kunstgriffe der Agenturtheorie}

Die Agenturtheorie stellt mit einem Kunstgriff - so Egon Franck (2011) - die Interessen der Aktionäre an die oberste Stelle (Jensen/Meckling 1976; Fama/Jensen 1983 a, 1983 b) und erstickt so die Diskussion um die Frage, wem das Residuum zusteht, bzw. postuliert wie selbstverständlich, dass es auf diese Frage nur eine Antwort geben kann: „Die Aktionäre sind die Prinzipale! “

Danach geht es in der Agenturtheorie nur noch darum, wie die Aktionäre ihre Interessen sichern und durchsetzen können. Da gemäss der Agenturtheorie die Aktionäre die einzigen Halter von Residualansprüchen sind, können nur sie zu Opfern eines schlechten bzw. eigennützigen Managements oder eines schwachen Managements und einer mächtigen und dreisten Arbeitnehmerschaft werden. Dementsprechend müssen den Aktionären wirksame Möglichkeiten des Durchgriffs in die Steuerung der Unternehmung eingeräumt werden. Deshalb ist es innerhalb dieses theoretischen Bezugsrahmens nur konsequent, primär Instrumente zu diskutieren, die der Sicherung der Interessen der Prinzipale, d. h. der Aktionäre, dienen.

Gemäss der Agenturtheorie entwickeln und nutzen Prinzipale verschiedene Instrumente, um ihre Interessen durchzusetzen. Sie verwenden Anreizsysteme (z. B. Aktienoptionen für das Topmanagement und für die Mitglieder des Boards (Verwaltungs- bzw. Aufsichtsrat; s. Hambrick/Jackson 2000)). Sie gestalten Strukturen (z. B. durch die Besetzung des 
Boards mit einer für sie vorteilhaften Mischung aus internen und externen Mitgliedern). Und sie nutzen bestehende Institutionen (Märkte). All das tun sie, um das Verhalten der Manager und Boardmitglieder (der Agenten) in ihrem Sinne zu disziplinieren. ${ }^{1}$ Das scheint jedoch nicht auszureichen. Deshalb wird gefordert, die Rechte der Aktionäre noch weiter zu stärken, um den Prinzipalen weitere Möglichkeiten des Durchgriffs in die Steuerung der Unternehmung zu ermöglichen (s. die Verweise in Franck 2011).

Bevor ich mit der Diskussion des Beitrages von Egon Franck fortfahre, erscheint es mir wichtig, noch eine weitere Setzung, d. h. einen weiteren Kunstgriff, der Agenturtheorie in Erinnerung zu rufen. Die Aktiengesellschaft ist in der Agenturtheorie lediglich ein Knotenpunkt von Verträgen, die zwischen Individuen geschlossen werden. Die Aktiengesellschaft, oder allgemeiner die Unternehmung oder die Organisation, wird als eine „legale Fiktion“ betrachtet:

„Contractual relations are the essence of the firm ... (M)ost organizations are simply legal fictions which serve as a nexus for a set of contractual relationships among individuals." (Jensen/Meckling 1976, 310)

Diese Definition der Organisation hat zwei entscheidende Konsequenzen. Zum einen verschwindet mit ihr die Unternehmung als korporativer Akteur, als eigenständige Rechtspersönlichkeit mit eigenen Zielen, aus der Organisationstheorie und der CorporateGovernance-Diskussion. Das heisst, die Unternehmung als analyserelevante Einheit löst sich mit dieser Setzung auf und der Blick richtet sich auf Verträge zwischen Individuen. Zum anderen verschwinden mit dieser Definition der Organisation auch die Elemente und die Funktionen der formalen Struktur von Organisationen, die etwa in der Bürokratietheorie (Weber 1972), in der Verhaltenswissenschaftlichen Entscheidungstheorie der Carnegie School (Simon 1949; March/Simon 1958; Cyert/March 1963) oder der Kontingenztheorie (s. zusammenfassend Kieser/Walgenbach 2010) im Vordergrund standen, aus der Diskussion über die Steuerung von Organisationen.

Die Agenturtheorie stellt mit ihren Setzungen und Kunstgriffen also nicht nur die Interessen der Prinzipale an die oberste Stelle, sondern sie blendet zugleich auch den korporativen Akteur, die Aktiengesellschaft als Rechtspersönlichkeit, aus.

\section{Die Agenturtheorie und die Wirklichkeit (Teil 1)}

Der Agenturtheorie bescheinigt Egon Franck (2011) eine gewisse Realitätsferne, distanziert sich zugleich in einer Fussnote (Fussnote 12) von der Agenturtheorie, zu deren Anhängern er sich längere Zeit selbst gezählt hat, und illustriert anschliessend in schlüssiger Weise anhand einiger Beispiele, wieso die Agenturtheorie die institutionelle Realität der Aktiengesellschaft nicht der Wirklichkeit entsprechend abbildet. Ich folge seiner Analyse in allen Punkten und könnte noch einige hinzufügen. Dazu gehört beispielsweise die oben erwähnte Betrachtung der Unternehmung als ein Knotenpunkt von Verträgen zwischen Individuen. Auch diese Basismetapher der Agenturtheorie hat nur wenig mit der institutionellen Realität der Unternehmung zu tun. Die Mitarbeiter der Daimler AG haben keinen Vertrag mit Herrn Dr. Zetsche und auch keinen Vertrag mit irgendeinem Klein- oder Gross-

1 In der Praxis waren es oftmals jedoch eher die Agenten, die diese Instrumente in ihrem Sinne umgesetzt und nutzbar gemacht haben (s. die Zusammenfassung der empirischen Befunde zur Agenturtheorie in Dalton et al. 2007). 
aktionär, sondern sie haben einen Vertrag mit der Daimler AG. „Die Aktiengesellschaft ist eine Gesellschaft mit eigener Rechtspersönlichkeit“, so steht es beispielsweise in $\mathbb{S} 1$ des deutschen Aktiengesetzes. Weiterhin lässt eine derartige auf das Individuum fokussierte Theorie ausser Acht, dass im Sinne der Agenturtheorie in vielen Organisationen nicht Individuen, sondern andere Organisationen (z. B. Banken, Versicherungen, Fondsgesellschaften) die Prinzipale sind. In diesem Sinne leben wir nicht nur in einer „market economy“, sondern auch in einer „organizational economy“ (Simon 1991).

\section{Eine Theorie ist eine Theorie! Mehr nicht!}

Was tun? Die Agenturtheorie komplett verwerfen? Nein, das wäre aus meiner Sicht der falsche Schritt. Die Agenturtheorie liefert m. E. ein begriffliches Instrumentarium, mithilfe dessen durch die Abstraktion von anderen in der Wirklichkeit relevanten Randbedingungen ein bestimmtes Problem, nämlich das Agenturproblem, in besonderer Weise hervorgehoben und verdeutlicht werden kann. Das ist ein wichtiger Beitrag! Sie liefert aber keine ausserhalb der theoretischen Modellierung verlässlich funktionierenden Lösungen für dieses Problem, weil sie eben von anderen relevanten Problemen abstrahiert und wichtige Randbedingungen ausblendet. Ist das schlimm? Nein! So ist Theorie nun einmal, so muss Wissenschaft (zumindest in den Sozialwissenschaften) nun einmal sein. Schlimm wird es, wenn Theorie mit Wirklichkeit verwechselt wird - man kann den Eindruck gewinnen, dass dies im Kontext der Agenturtheorie schon öfter geschehen ist - und (ver)einfache(nde) Lösungen aus abstrahierenden Theorien abgeleitet und umgesetzt werden.

\section{Die Agenturtheorie und die Wirklichkeit (Teil 2)}

Was aber nun tun? Zunächst einmal das, was Egon Franck in seinem Beitrag leistet, und das heisst, genauer hinschauen, die polit-ökonomischen Grundannahmen der Theorie mit der polit-ökonomischen Wirklichkeit vergleichen, die institutionellen Grundkonzepte der Theorie mit der institutionellen Wirklichkeit konfrontieren, danach fragen, wie es praktisch, da draussen in der „wirklichen“ Welt, aussieht.

$\mathrm{Zu}$ Recht weist Egon Franck zunächst mit Verweis auf Stout (2007) darauf hin, dass auch Mitarbeiter, Lieferanten, Kreditgeber, Kunden und andere Stakeholder ${ }^{2}$ Ressourcen wie Zeit, Mühe, Wissen und Geld in Unternehmungen einbringen. Diese Investitionen sind nur dann von Wert, wenn die Unternehmung nicht untergeht, die Beziehungen von Dauer sind und die Schäden, die von der Unternehmung bspw. durch den Verzehr oder die Übernutzung rein öffentlicher Güter hervorgerufen werden, den Nutzen, der aus der Existenz der Unternehmung gezogen wird, nicht überkompensieren. Unternehmensspezifisches $\mathrm{Hu}-$ mankapital beispielsweise ist im Falle des Untergangs einer Unternehmung wertlos. Unverschuldet entlassene Mitarbeiterinnen und Mitarbeiter müssen mit deutlichen Gehaltsabschlägen rechnen (Franck 2011). Insofern tragen nicht nur die Aktionäre, sondern auch die Mitarbeiterinnen und Mitarbeiter ein erhebliches Risiko, das an die Entwicklung und den Fortbestand der Unternehmung gebunden ist.

Weiterhin zeigt Egon Franck durch die Bezugnahme auf bestehende Gesetze und seine Verweise auf Arbeiten von Autoren, die wie er damit begonnen haben, die Agenturtheorie mit der institutionellen Wirklichkeit zu kontrastieren, dass unabhängig von nationalen Re-

2 Wie z. B. zunehmend auch der Steuerzahler. 
gulierungsunterschieden die Durchgriffsmöglichkeiten der Aktionäre gering sind: „Das Gesetz weist nämlich unmissverständlich dem Board of Directors - dem Verwaltungsrat (in der Schweiz, P.W.) - die zentrale Entscheidungsgewalt in der Aktiengesellschaft zu“ (Franck 2011, 203). In den USA ist dies nicht grundsätzlich anders (Bainbridge 2006) und in anderen Ländern auch nicht (Franck 2011).

Nicht zu Unrecht halten deshalb auch andere Autorinnen und Autoren wie Lan/Heracleous (2010, 301) dem zentralen Argument der Agenturtheorie, dass die Anteilseigner Eigentümer der Unternehmung sind und deshalb den Anspruch auf das Residuum und ein Vorrecht gegenüber allen anderen Anspruchsgruppen haben, entgegen:

"This argument is based on a misinterpretation of the legal position on the issue of ownership, and ignores the legal principles of personification of the corporation, of limited liability of shareholders and of personal, potential unlimited liability of directors."

Und Blair/Stout $(2001,423)$ problematisieren in der folgenden Aussage die Metapher des Knotenpunktes von Verträgen zwischen Individuen noch weit stärker:

„When a corporation is formed, a new legal entity is born. As a matter of law, this entity, and not any of its participants, owns the assets used in corporate production, as well as any surplus produced by the enterprise."

\section{Das „Board Primacy“-Modell als Alternative}

Was aber nun tun? Wieder das, was auch Egon Franck in seinem Beitrag begonnen hat: Alternativen diskutieren! Und er tut dies, indem er die oben diskutierten Ausblendungen der Agenturtheorie schrittweise wieder einblendet.

Zunächst argumentiert Egon Franck (2011), dass wenn schon die Position der Aktionäre gestärkt werden soll, dies nur Hand in Hand mit einer Stärkung der Position der Mitarbeiterinnen und Mitarbeiter erfolgen sollte, die wie die Aktionäre Risiken tragen und in deren Löhne und Gehälter Teile des Residuums einfliessen. An die Stelle des in den meisten Ländern als unabhängig konzipierten Boards (Verwaltungs- bzw. Aufsichtsrat) müsste nach dieser Sichtweise eine Art Interessenvertretergremium treten, das aus Delegierten der Aktionäre und der Mitarbeiterinnen und Mitarbeiter besteht. Aber schon im nächsten Abschnitt problematisiert Egon Franck diese Lösung, in dem er darauf hinweist, dass ein mit Interessenvertretern besetztes Gremium dazu neigt, sich in eine Arena kostenintensiver interessengeleiteter Rentensuche zu verwandeln, insbesondere wenn die Interessensdivergenzen der vertretenen Stakeholdergruppen erheblich sind. Er problematisiert diese Lösung zusätzlich durch den Hinweis, dass auch innerhalb der einzelnen Stakeholdergruppen Interessensdivergenzen bestehen können und tatsächlich oft auch bestehen. ${ }^{3}$ Deshalb droht so Egon Franck (2011) - ein mit Interessenvertretern besetztes Führungsgremium der Aktiengesellschaft zur Arena der Rentensuche mit eskalierenden Politikkosten zu werden.

In seinem zweiten Schritt schliesst sich Egon Franck deshalb der u. a. von Blair/Stout (1999, 2001; sowie Stout 2003) vertretenen Position einer „Board Primacy“ an, welche in jüngerer Zeit auch zunehmend an prominenter Stelle in der internationalen Organisations-

3 Das hier skizzierte Problem verstärkt sich noch dadurch, dass sich ähnliche Argumente, wie sie mit Blick auf die Stärkung der Aktionärs- bzw. der Mitarbeiterrechte vorgetragen wurden, für viele weitere Stakeholdergruppen anführen lassen. 
und Managementliteratur diskutiert wird (s. bspw. Lan/Heracleous 2010). Die Position der „Board Primacy“ bedeutet die Zurückdrängung der Interessen der unterschiedlichen Stakeholdergruppen. Das Board (der Verwaltungs- bzw. Aufsichtsrat) soll gemäss dieser Perspektive als unabhängiger Treuhänder agieren. Egon Franck folgt damit einer Betrachtungsweise, die in den meisten Ländern der gesetzlichen Lage entspricht, und stellt sich dezidiert (s. Fussnote 12 in Franck 2011) gegen die Argumente einer agenturtheoretisch fundierten Fassung der Corporate Governance, die eine „Shareholder Primacy“ impliziert. Er tut dies, diesen Eindruck vermitteln die einleitenden und abschliessenden Zeilen seines Beitrags, weil er - wie viele andere auch (s. exemplarisch Davis 2009; Felin/Foss 2009; Dobbin/Jung 2010) - einen engen Zusammenhang zwischen der Umsetzung der Konzepte der normativen Agenturtheorie und den Aufsehen erregenden Unternehmensskandalen der letzten Jahre (Stichwort Enron) sowie der jüngsten Finanzkrise sieht.

Auch diesen Schritt gehe ich mit Egon Franck und stimme seiner Beantwortung der von ihm aufgeworfenen Frage zu. Die Aktionärsrechte zu stärken, das wäre - vor dem Hintergrund des derzeitigen Wissensstands - vermutlich der falsche Schritt. Im Gegenteil, vor dem Hintergrund einer inzwischen umfangreichen empirischen Evidenz (s. zusammenfassend Dalton et al. 2007), dass die Instrumente und Vorschläge der Agenturtheorie oftmals das Gegenteil dessen bewirkt haben, was mit ihnen intendiert war (Dobbin/Jung 2010; s. hierzu auch den bemerkenswerten Beitrag von Jensen et al. 2004), spricht sogar vieles dafür, die im Kontext einer von der Agenturtheorie dominierten Corporate-Governance-Debatte eingeführten und verstärkt genutzten Steuerungsinstrumente, wie insbesondere die erfolgsabhängige Entlohnung des Topmanagements und des Boards durch Aktienoptionen, ausser Kraft zu setzen oder zumindest wieder deutlich zurückzunehmen.

\section{Gibt es weitere Alternativen? Der mögliche Beitrag der Betriebswirtschaftslehre}

Vorwärts, rückwärts - diese Schritte bin ich nun mit Egon Franck gegangen. Aber was jetzt? Stehen bleiben? Nein! Mein Vorschlag mit Blick auf die Wirtschaftswissensschaften lautet: Zunächst seitwärts! Nicht gleich zum alten, sich in den geltenden Gesetzen spiegelndem Modell der „Board Primacy“ zurückkehren und nun voll auf dieses setzen, sondern neue und alternative Modelle entwickeln und diskutieren. Ein alternatives Modell wird etwa von Lan/Heracleous (2010) vorgeschlagen. Ihr Modell setzt am „Board Primacy"-Modell an, stellt aber den alle Stakeholder verbindenden korporativen Akteur explizit an die oberste Stelle der Pyramide der berechtigten Interessen in und an der Unternehmung. Nicht die Gruppe der Shareholder und auch nicht irgendeine andere Anspruchsgruppe wird in ihrem Modell als Prinzipal betrachtet, sondern die Unternehmung, der korporative Akteur selbst, wird zum Prinzipal gemacht. Dieser Vorschlag von Lan/Heracleous erscheint mir - trotz aller Kritik, die sich anbringen lässt (s. Walgenbach 2011) - zumindest diskussionswürdig, zumal er noch deutlicher als das „Board Primacy“-Modell werden lässt, dass - wie in den gesetzlichen Regelungen in vielen Ländern vorgesehen - die Organe der Aktiengesellschaft nicht im Interesse irgendeiner Anspruchsgruppe, sondern im Interesse der Unternehmung handeln sollen. Zum Seitwärtsschritt gehört es dann weiterhin, darüber nachzudenken, welche Beiträge die Betriebswirtschaftslehre zur Ausdifferenzierung dieses Modells leisten könnte, wenn es denn als ein für die Betriebswirtschaftslehre relevantes Modell erachtet wird.

Weitere Seitwärtsschritte wären darin zu sehen, dass systematisch komparative Studien, und zwar auf der Basis unterschiedlichster Methoden durchgeführt werden, um die unter- 
schiedlichen bereits entwickelten und noch zu entwickelnden Modelle der Aktiengesellschaft im Speziellen und der Organisation im Allgemeinen zu testen. $\mathrm{Zu}$ den unterschiedlichen Methoden der Theorien- und Konzeptgenerierung gehört auch „armchair theorizing “, weil auch neue (bisher nicht existente) Steuerungskonzepte entwickelt werden könnten und sollten. $\mathrm{Zu}$ den unterschiedlichen Methoden des vergleichenden Testens gehören z. B. Simulationsstudien, Systems Thinking und Systems Dynamics sowie Laborexperimente, ${ }^{4}$ um nur einige zu nennen. Zu den mir sinnvoll erscheinenden komparativen Studien gehört auch der systematische Vergleich der bestehenden institutionellen Regelungen in unterschiedlichen Ländern und ihrer jeweiligen Auswirkungen. Solche Analysen liegen bisher erst in Grundzügen vor (Lubatkin et al. 2005; Aguilera et al. 2008; Aguilera/Jackson 2010).

Sind diese Schritte getan, müsste es „stehen“ heissen. Innehalten! Reflektieren, um dann die nächsten auf das Ziel einer guten, d. h. wirksamen, und von den unterschiedlichen Stakeholdern akzeptierbaren Corporate Governance gerichteten (normativen) Schritte zu tun. Diese weiteren Schritte, insbesondere jene Schritte, die normative Aussagen implizieren, sollten erst im Anschluss an die vergleichenden Analysen getan werden. Normative Aussagen müssen noch nicht einmal aus den Analysen folgen. Eine klare Trennung zwischen Sein und Sollen ist wichtig! Anderenfalls würde diese neue Forschungsrichtung in der Betriebswirtschaftslehre schnell denselben Versuchungen anheimfallen wie die Agenturtheorie.

\section{Schlussfolgerung}

Wenn die Agenturtheorie wirklich ein Irrweg ist bzw. die Umsetzung ihrer normativen Implikationen Unternehmungen und vielleicht inzwischen sogar die gesamte Gesellschaft in nicht wünschenswerte Richtungen führt, worauf die vorliegende und inzwischen umfangreiche empirische Evidenz hindeutet (s. Dalton et al. 2007 und die dort zusammengetragene Literatur), dann sollten wir als Wirtschaftswissenschaftlerinnen und Wirtschaftswissenschaftler mit unseren Mitteln handeln, so wie es Egon Franck (2011) beispielsweise mit seinem Aufsatz getan hat.

\section{Literaturhinweise}

Aguilera, R.V./Filatotchev, I./Gospel, H./Jackson, G. (2008): An organizational approach to comparative corporate governance: Costs, contingencies, and complementarities, in: Organization Science, Jg. 19, S. 475-492.

Aguilera R.V.JJackson, G. (2010): Comparative and international corporate governance, in: Academy of Management Annals, Jg. 4, S. 485-556.

Bainbridge, S.M. (2006): Director primacy and shareholder disempowerment, in: Harvard Law Review, Jg. 119, S. 1735-1758.

Blair, M.M./Stout, L.A. (1999): A team production theory of corporate law, in: Virginia Law Review, Jg. 85, S. 247-328.

4 Diese Methoden dienen der Analyse komplexer Systeme und Zusammenhänge. Es sind Experimente, die zunächst ohne Konsequenzen in der Praxis bleiben. Dies ist wichtig, da eine Einführung bestimmter neuer Regelungen in die Praxis in der Zukunft weitreichende Folgen nach sich ziehen kann, die es zuvor abzuschätzen gilt. 
Blair, M.M./Stout, L.A. (2001): Director accountability and the mediating role of the corporate board, in: Washington University Law Quarterly, Jg. 79, S. 403-447.

Cyert, R.M./March, J.G. (1963): A Behavioral Theory of the Firm, Cambridge, MA.

Dalton, D.R./Hitt, M.A./Certo, S.T./Dalton, C.M. (2007): The fundamental agency problem and its mitigation: Independence, equity, and the market for corporate control, in: Academy of Management Annals, Jg. 1, S. 1-64.

Davis, G.F. (2009): Managed by the Markets: How Finance Re-Shaped America, Oxford.

Dobbin, F./Jung, J. (2010): The misapplication of Mr. Michael Jensen: How agency theory brought down the economy and why it might again, in: Lounsbury, M./ Hirsch, P.M. (Hrsg.): Markets on Trial: The Economic Sociology of the U.S. Financial Crisis: Part B, Research in the Sociology of Organizations, Jg. 30, Bingley, S. 29-64.

Fama, E.F./Jensen, M.C. (1983 a): Separation of ownership and control, in: Journal of Law and Economics, Jg. 26, S. 301-325.

Fama, E.F./Jensen, M.C. (1983 b): Agency problems and residual claims, in: Journal of Law and Economics, Jg. 26, S. 327-349.

Felin, T./Foss, N.J. (2009): Social reality, the boundaries of self-fulfilling prophecy, and economics, in: Organization Science, Jg. 20, S. 654-668.

Franck, E. (2011): Ist es an der Zeit, die Aktionärsrechte zu stärken?, in: Die Unternehmung, Jg. 65, S. 201-214.

Hambrick, D.C./Jackson, E.M. (2000): Outside directors with a stake: The linchpin in improving governance, in: California Management Review 42, S. 108-127.

Jensen, M.C./Meckling, W.F. (1976): Theory of the firm: Managerial behaviour, agency costs, and ownership structure, in: Journal of Financial Economics, Jg. 3, S. 305-360.

Jensen, M.C./Murphy, K.C./Wruck, E.G. (2004): Remuneration: Where we've been, how we got to here, what are the problems, and how to fix them, ECGI Working Paper No. 44/2004, ECGI Working Paper Series in Finance.

Kieser, A./Walgenbach, P. (2010): Organisation, 6. Aufl., Stuttgart.

Lan, L.L./Heracleous, L. (2010): Rethinking agency theory: The view from law, in: Academy of Management Review, Jg. 35, S. 294-314.

Lubatkin, M.H./Lane, P.J./Collin, S.O./Very, P. (2005): Origins of Corporate Governance in the USA, Sweden and France, in: Organization Studies, Jg. 26, S. 867-888.

March, J.G./Simon, H.A. (1958): Organizations, New York.

Simon, H.A. (1949): Administrative Behavior: A Study of Decision-Making Processes in Administrative Organizations, New York.

Simon, H.A. (1991): Organizations and markets, in: Journal of Economic Perspectives, Jg. 5, S. 25-44.

Stout, L.A. (2003): The shareholder as Ulysses: Some empirical evidence on why investors in public corporations tolerate board governance, in: University of Pennsylvania Law Review, Jg. 152, S. 1-47.

Stout, L.A. (2007): The mythical benefits of shareholder control, in: Virginia Law Review, Jg. 93, S. 789-809.

Walgenbach, P. (2011): Das Ende der Organisationsgesellschaft und die Wiederentdeckung der Organisation, in: Die Betriebswirtschaft, Jg. 71, S. 443-462.

Weber, M. (1972): Wirtschaft und Gesellschaft, 5. Aufl., Tübingen. 
Peter Walgenbach, Dr., ist Professor für Betriebswirtschaftslehre/Organisation, Führung und Human Resource Management an der Friedrich-Schiller-Universität Jena.

Anschrift: Friedrich-Schiller-Universität Jena, Wirtschaftswissenschaftliche Fakultät, CarlZeiß-Straße 3, D-07743 Jena, Tel.: +49 (0) 36419-43130, E-Mail: peter.walgenbach@unijena.de 